ar it bureciu of Standards

Lwary, N.... Blag

OCT 111965

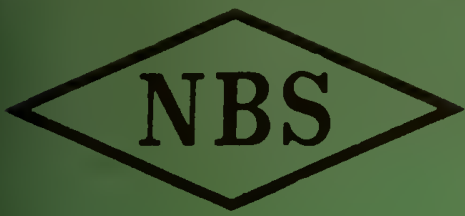

Eechnical Note

$\eta_{0.323}$

\title{
REFRACTIVE INDEX AND DISPERSION OF LIQUID HYDROGEN
}

R. J. CORRUCCINI

U. S. DEPARTMENT OF COMMERCE

NATIONAL BUREAU OF STANDARDS 


\section{THE NATIONAL BUREAU OF STANDARDS}

The National Bureau of Standards is a principal focal point in the Federal Government for assuring maximum application of the physical and engineering sciences to the advancement of technology in industry and commerce. Its responsibilities include development and maintenance of the national standards of measurement, and the provisions of means for making measurements consistent with those standards; determination of physical constants and properties of materials; development of methods for testing materials, mechanisms, and structurcs, and making such tests as may be necessary, particularly for government agencies; cooperation in the establishment of standard practices for incorporation in codes and specifications; advisory service to government agencies on scientific and technical problems; invention and development of devices to serve special needs of the Government; assistance to industry, business, and consumers in the development and acceptance of commercial standards and simplified trade practice recommendations; administration of programs in cooperation with United States business groups and standards organizations for the development of international standards of practice; and maintenance of a clearinghouse for the collection and dissemination of scientific, tech. nical, and engineering information. The scope of the Bureau's activities is suggested in the following listing of its four Institutes and their organizational units.

Institute for Basic Standards. Applied Mathematics. Electricity. Metrology. Mechanics. Heat. Atomic Physics. Physical Chemistry. Laboratory Astrophysics. * Radiation Physics. Radio Standards Laboratory:" Radio Standards Physics; Radio Standards Engineering. Office of Standard Reference Data.

Institute for Materials Research. Analytical Chemistry. Polymers. Metallurgy. Inorganic Materials. Reactor Radiations. Cryogenics.* Materials Evaluation Laboratory. Office of Standard Reference Materials.

Institute for Applied Technology. Building Research. Information Technology. Performance Test Development. Electronic Instrumentation. Textile and Apparel Technology Center. Technical Analysis. Office of Weights and Measures. Office of Engineering Standards. Office of Invention and Innovation. Office of Technical Resources. Clearinghouse for Federal Scientific and Technical Information. **

Central Radio Propagation Laboratory.* Ionospheric Telecommunications. Tropospheric Telecommunications. Space Environment Forecasting. Aeronomy.

* Located at Boulder, Colorado 80301.

** Located at 5285 Port Royal Road, Springfield, Virginia 22171. 


\title{
NATIONAL BUREAU OF STANDARDS Eechnical Note 323
}

ISSUED September 24, 1965

\section{REFRACTIVE INDEX AND DISPERSION OF LIQUID HYDROGEN}

\author{
R. J. Corruccini \\ Cryogenics Division \\ Institute for Materials Research \\ National Bureau of Standards \\ Boulder, Colorado
}

NBS Technical Notes are designed to supplement the Bureau's regular publications program. They provide a means for making available scientific data that are of transient or limited interest. Technical Notes may be listed or referred to in the open literature.

\footnotetext{
For sale by the Superintendent of Documents, U. S. Government Frinting Office

Washington, D.C. 20402

Price: $25 \$$
} 
Abstract

1. Introduction

2. Dispersion

Table 1 - Refractive Indices of Hydrogen Gas at STP --- 5

3. Experimental Refractive Indices of the Liquid - 6

3. 1. Data of Belonogov and Gorbunkov [1] Table 2 - Dispersion of Hydrogen -.-_-_--- 8

3.2. Data of Johns and Wilhelm [2]

3.3. Data of Augustin [3]

3.4. Data of Scheel [20]

3.5. Summary and Comparison of the Data

4. Calculation of Refractive Index from Dielectric

Constant -

Table 3 - Summary of deviations, $10^{4}$ ( $\left._{\text {calc. }}{ }^{-n_{\text {exptl. }}}\right)-14$

5. The Ortho vs Para Difference $\ldots$

6. Proposed Experimental Method _. 17

7. Symbols

8. References -

Figure 1. Specific refraction of liquid hydrogen as a function of wavelength

Figure 2. Specific refraction of hydrogen plotted according to Cauchy dispersion formula _......... 22 

Refractive Index and Dispersion of Liquid Hydrogen

R. J. Corruccini

The published experimental values of the refractive index of liquid hydrogen have been correlated by calculating and comparing the values of the Lorentz-Lorenz ratio as a function of wavelength. Because of the scatter found by this analysis, it is proposed that more accurate values can be calculated from available data of other kinds, namely, the dielectric constants determined by Stewart and the optical dispersion determined for the gas at STP. Formulas for this calculation are given.

Key Words: Dispersion, hydrogen, index of refraction, liquid, optical, refractive index

\section{Introduction}

The refractive index of liquid hydrogen is of interest at present in two kinds of practical application: (a) the optics of bubble chambers, and (b) as a prospective tool for densitometry. There have been several determinations $[1,2,3]$. These have been made by what may be called deviation methods, i.e., methods based on determination of the bending of a light beam on passage between a reference medium and the fluid sample. From the precision of these data and the agreement among the different investigations it appears that these results are not more accurate than a few hundredths of one percent in the refractive index.

Independent evidence that the se data are of relatively low precision can be obtained by contrasting them with recent measurements of 
the related property, the dielectric constant, by Stewart [4]. Stewart found a weak dependence of the polarization or Clausius-Mossotti function,

$$
p \equiv \frac{\epsilon-1}{\rho(\epsilon+2)}
$$

upon the density. The maximum variation of the polarization between zero density and higher densities was about $0.2 \%$, and the variation of the polarization between the densities of the triple point and the boiling point was $0.05 \%$. Since the specific polarization can be identified with the specific refraction or Lorentz-Lorenz function,

$$
r_{\lambda} \equiv \frac{n^{2} \lambda-1}{\rho\left(n_{\lambda}^{2}+2\right)}
$$

via the dispersion and Maxwell's relation,

$$
\epsilon=\mathrm{n}_{\infty}^{2},
$$

which is equivalent to

$$
p=r_{\infty},
$$

one would expect the specific refraction also to show this dependence on density. However, in those investigations that covered a range of densities $[1,2]$, such an effect is lost in the scatter of the calculated refractions. Hence we conclude that their accuracy and precision are

\footnotetext{
See sec. 7 for definitions of symbols.
} 
considerably poorer than those of Stewart.

Stewart claimed a precision of $0.05 \%$ in $€-1$ which corresponds to about $0.005 \%$ in $\mathrm{n}_{\infty}$ of the liquid. It is suggested here that values of $\mathrm{n}_{\lambda}$ calculated from Stewart's dielectric constant data may be more accurate than the available experimental values. In order to complete such a calculation, a dispersion relation is needed,

$$
r_{\lambda}-r_{\infty}=f(\lambda)
$$

A crude relation is derivable from the experimental data on the liquid, and a much more precise one is derivable from available data on the gas at STP. The latter data determined by interferometry are as much as two orders of magnitude more precise in $r$. Therefore it is suggested that a dispersion relation obtained from the data on the gas is to be preferred even though it is the liquid state for which we wish to make predictions. As will be shown, the dependence of $r$ on density and temperature is small. Hence the gaseous dispersion corrections should be sufficiently accurate regardless of state.

In this note we will examine the experimental data, then will construct formulas for calculating $\mathrm{n}_{\lambda}$ from Stewart's dielectric constants, and finally will compare the two kinds of results. It is necessary to begin with the dispersion of the gas, as this enters into the discussion of some of the experimental refractive indices of the liquid.

\section{Dispersion}

The Landolt-Börnstein tables [5] give extracts of much of the published data on gaseous hydrogen. Additional rếerences are given in a compilation by Maryott and Buckley [6]. There are five sources of data in the visible and ultraviolet, of which four are in excellent agreement: Cuthbertson and Cuthbertson [7], Koch [8], Kirn [9], and Tausz 
and Görlacher [10]. (The data of Frivold, Hassel, and Rustad [11] after correcting to the real gas are distinctly lower.) The excellence of this agreement is shown by the summary of values of $n_{\infty}^{2}-1$ given by Maryott and Buckley. (The extrapolation to $\lambda=\infty$ was made in each case by using the original authors' own dispersion formulas.) The four sources mentioned above gave values of $10^{6}\left(\mathrm{n}_{\infty}-1\right)$ ranging from 136.1 to 136.4. In good agreement with these are two measurements that essentially gave $\mathrm{n}_{\infty}$ directly by operating at wavelengths so great that $\mathrm{n}$ had practically attained its limiting value. These are (a) measurements at 6.7 and 8.7 microns in the infrared by Koch [8], both giving $10^{6}\left(\mathrm{n}_{\infty}-1\right)$ = 136. 1, and (b) a microwave determination by Essen [12] at $9000 \mathrm{Mc} / \mathrm{s}$ giving 136.0 \pm 0.2 . From this body of consistent data we will obtain a representation of the dispersion of hydrogen.

For this purpose, only values of $n$ calculated by the formulas of Koch and of Kirn will be used, the reasons being as follows: (a) These authors made much more extensive measurements than the others. Koch determined $n$ at twenty wavelengths from 2302 to $5461 \AA$. Kirn determined $\mathrm{n}$ at fifteen wavelengths from 1854 to $5461 \AA$. Thus it is felt that their dispersion relations ought to be more accurate than those of Cuthbertson and of Tausz, each of whom used only four wavelengths and employed simpler formulas. (b) The agreement between Koch and Kirn is excellent, as is shown in Table 1. Neither of the se authors agrees as well with Cuthbertson or Tausz as they do with each other. 
Table 1

Refractive indices of hydrogen gas at STP

\begin{tabular}{|l|llcc|}
\cline { 3 - 5 } \multicolumn{1}{c|}{} & \multicolumn{4}{|c|}{$10^{6}\left(\mathrm{n}_{\lambda}-1\right)$} \\
\hline Source & 4358 & 5461 & 6939 & $\infty$ \\
\hline Koch, [8] & 141.794 & 139.660 & $(138.277)$ & $(136.101)$ \\
Kirn, [9] & 141.739 & 139.628 & $(138.220)$ & $(135.990)$ \\
\hline
\end{tabular}

Note: The values were calculated from the respective dispersion formulas of Koch and Kirn. The parenthetic values are extrapolations.

Various formulas have been used for representing dispersion, all being derived from the fundamental relation [13]:

$$
r_{\lambda}=a \sum_{i} \frac{b_{i}}{\nu_{i}^{2}-\nu^{2}}
$$

in which one or two terms are used, the constants, $a, b_{i}$, and $\nu_{i}$ being evaluated from the data. For the present purpose a convenient formula is obtained by expansion of each term, collection of like terms, and truncation after the term in $\nu^{4}$, thus:

$$
r_{\lambda}=r_{\infty}+\frac{A}{\lambda^{2}}+\frac{B}{\lambda^{4}}
$$

This permits the density dependence to be separated from the dispersion in the term, $r_{\infty}$; i.e., the coefficients, $A$ and $B$, are treated as independent of density. Equation (7) was fitted to the average of the smoothed refractive indices of Koch and Kirn at the two mercury lines used in nearly all of the various investigations, 4358 and $5461 \AA$, and at $\lambda=\infty$. 
This gave $A=0.7799569 \times 10^{6}$ and $B=0.495126 \times 10^{12}$ for $\lambda$ in $\AA$ and $r$ in $\mathrm{cm}^{3} / \mathrm{g}$. Since this procedure fits an average of two sets of smoothed data with a function that behaves like the original smoothing functions, it necessarily follows a course between the original functions. The average deviation from those experimental points of Koch and Kirn lying in the visible region is $0.04 \%$. The formula is not intended for use at $\lambda<4000 \AA$.

3. Experimental Refractive Indices of the Liquid 3. 1. Data of Belonogov and Gorbunkov [1]

These authors determined the refractive index of saturated liquid parahydrogen from about the nbp (normal boiling point) to $30.5^{\circ} \mathrm{K}$ and at the wavelengths, 4360, 5460, and $5790 \AA$. They also determined the refractive index of saturated liquid normal hydrogen over the same temperature range but at $5460 \AA$ only. Their temperature range meets that of the other investigators at about the nbp but does not overlap it. They are the only authors to have measured the refractive index of parahydrogen or the para-normal difference. The following comments may be made about their work:

(a) Their claimed accuracy is $\pm 2 \times 10^{-4}$ in the absolute values of $\mathrm{n}$ and $\pm 1 \times 10^{-4}$ in the difference of refractive index between normal and parahydrogen. An image displacement method was used.

(b) Their data are presented in small graphs. I estimate the error in reading values of $\mathrm{n}$ from these graphs by an expandable scale and magnifier to be about $\pm 2 \times 10^{-4}$. This must be combined with their estimated experimental error of the same magnitude to give an overall error ( $\mathrm{rms}$ ) of the recovered data of about $\pm 3 \times 10^{-4}$. This corresponds to about $\pm 0.3 \%$ in $r$. They present the data for both forms of hydrogen at $5460 \AA$ in one graph 
as a function of temperature and in another graph as a function of density. The densities were obtained from the data of Goodwin et al. [14]. It should be possible to estimate the graph-reading error by comparing the results read from these two graphs. On comparing specific refractions calculated from the data of the two graphs a consistent difference of about $0.3 \%$ was found. This corresponds to $3 \times 10^{-4}$ in $\mathrm{n}$. There was little overlap of the two sets. Thus it appears that the estimate above of reading error is conservative, but that detectable systematic inconsis tencies exist between the different representations of the same data in the paper of Belonogov and Gorbunkov.

(c) The authors state that their results "correspond to the $\mathrm{L}-\mathrm{L}$ formula with an accuracy of $5 \times 10^{-5}$." The meaning of this statement is obscure. The most conservative interpretation would seem to be that $r$ is constant within limits equivalent to $\pm 5 \times 10^{-5}$ in the refractive index. However, the author ${ }^{\prime}$ claimed accuracy in $n$ was only $\pm 2 \times 10^{-4}$. Also, in order to obtain such a finding, the density would have to be known more accurately than 1 part in 2000. This is not the case [14]. Thus it seems that these authors could not possibly have known either $\mathrm{n}$ or $\rho$ accurately enough to support the statement quoted above. Nevertheless, the L-L relation seems to be obeyed, inasmuch as I find no consistent dependence of their values of $r$ on $\rho$. However, in comparing the values of $r$ for a particular form of hydrogen at a particular wavelength, variations from the mean as large as $0.5 \%$ were found indicating that the error estimate in (b) above is too small.

(d) Because of the apparent applicability of the L-L formula, all of the results at a given wavelength for a given form of hydrogen 
can be lumped together in an average value of $r_{\lambda}$, independently of the temperatures and pressures at which the individual measurements of $n$ were made. The dispersion then $c$ an be expressed simply as the dependence of $r_{\lambda}$ on $\lambda$. The average values of $r_{\lambda}$ for parahydrogen at the three wavelengths are shown in figure 1 and Table 2.

\section{Table 2}

Dispersion of hydrogen

\begin{tabular}{|c|c|c|c|c|c|}
\hline$\lambda, \AA$ & \multicolumn{5}{|c|}{ Experimental values of $\mathrm{r}_{\lambda}, \mathrm{cm}^{3} / \mathrm{g}$} \\
\hline \multirow{4}{*}{$\begin{array}{l}4047 \\
4358-60^{\dagger}\end{array}$} & $\mathrm{B} \& \mathrm{G}[1]$ & ] liquid & $\mathrm{J} \& \mathrm{~W}[2]$ liquid & Aug. [3] liquid & $\mathrm{K}-\mathrm{K}_{\text {, * }}$ gas STP \\
\hline & para & normal & normal & normal & normal \\
\hline & & \multirow{7}{*}{ 1. 038} & \multirow{4}{*}{$\begin{array}{l}1.050 \\
1.032\end{array}$} & 1.0556 & \multirow{5}{*}{$\begin{array}{l}1.0508 \\
1.0350\end{array}$} \\
\hline & 1.056 & & & 1.0480 & \\
\hline $5460-61^{\dagger}$ & 1.032 & & & 1.0319 & \\
\hline 5790 & 1.026 & & & 1.0292 & \\
\hline 6563 & & & \multirow{3}{*}{$\begin{array}{c}1.018_{5} \\
(0.998)\end{array}$} & 1.0246 & \\
\hline 6939 & & & & & \multirow[b]{2}{*}{$(1.0083)$} \\
\hline$\infty$ & $(0.988)$ & & & $(1.0106)$ & \\
\hline $10^{6} \mathrm{~A}$ & 1.301 & & 0.984 & 0.5165 & 0.7800 \\
\hline $10^{12} \mathrm{~B}$ & & & & 3.632 & 0.4951 \\
\hline Av. Dev. & 0.00037 & & 0.00043 & 0.00009 & \\
\hline
\end{tabular}

* See sec. 2 for origins of this column.

$\dagger$ This is the range of nominal values for this $\mathrm{Hg}$ line quoted by the various authors.

* Average absolute difference between $r_{\lambda}$ calculated by the dispersion formulas and the experimental values. 
If these data are fitted by the Cauchy formula (7), further evidence of imprecision is found, namely that the coefficient $B$ is negative. This is readily seen if $r_{\lambda}$ is plotted versus $1 / \lambda^{2}$ as in figure 2 ; i.e. , a simple curve through the three points of $B-G$ is concave downwards. However, for the behavior to be physically realistic, both of the coefficients, $A$ and $B$, must be positive, giving a dispersion curve of the shape shown schematically by the solid curve in the inset figure. This behavior, corresponding to equation (6), consists of an asymptotic decrease to a limiting value, $r_{\infty}$, at zero frequency and a rise towards infinity at frequencies $\nu_{i}$, in the ultraviolet, corresponding to electronic resonances of the molecule. The negative $B$ coefficient does not have much effect on the extrapolation to $\lambda=\infty$ but results in negative

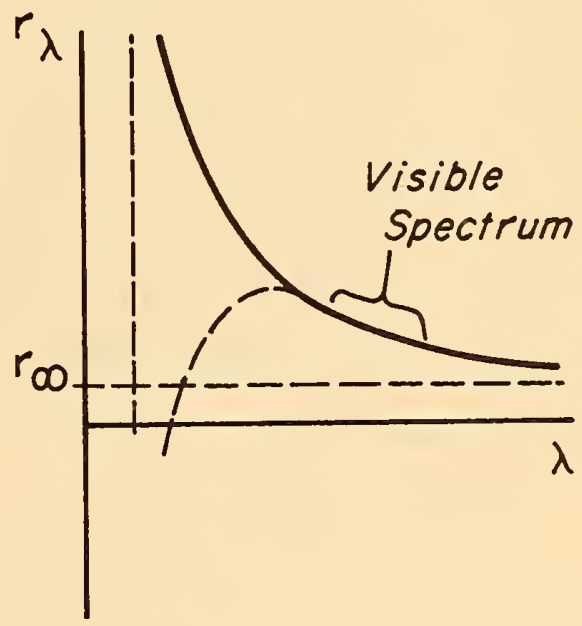
values at short wavelengths (dotted curve in inset figure); this is the physically unreal behavior just referred to. Obviously then, the dispersion formula must be truncated at the term in $1 / \lambda^{2}$. The values of $r_{\infty}$ and $\mathrm{A}$ in Table 2 were obtained by least-squares fitting of the truncated formula to the three values of $\mathbf{r}_{\lambda}$.

\subsection{Data of Johns and Wilhelm [2]}

These authors made measurements on normal hydrogen from the triple point to the nbp at the wavelengths 4358,5461 , and $6939 \AA$. The specific refractions, as calculated by them using the densities of Kamerlingh Onnes and Crommelin [15], showed no dependence on temperature or density. These have been recalculated using modern density data. The correlation for normal hydrogen by Goodwin et al. [14] 
has been adopted for this purpose. The latter densities are from 0.1 to $0.2 \%$ higher than the older values. Their use does not alter the conclusion that Johns and Wilhelm's specific refractions are independent of temperature and density. The net effect is to lower their average values of $r_{\lambda}$ by 0.16 to $0.18 \%$.

An attempt was made to adjust the temperature scale used by Johns and Wilhelm to a modern basis. The only clue to what their scale was is their statement that the nbp of hydrogen is $20.41^{\circ} \mathrm{K}$. By referring to a listing by Moessen, Aston, and Ascah [16] of the older determinations of the $\mathrm{nbp}$, we find that there was a Physikalische Technische Reichsanstalt scale [17] on which the nbp of hydrogen could be construed to have this value. If this were the one used, it would be reasonable to correct all of Johns and Wilhelm's temperatures by the factor 20.38/20.41. (20.38 is taken to be the nbp of $n-\mathrm{H}_{2}$ on the NBS-1955 scale.) I have rather arbitrarily chosen to do this. Another possibility is that Johns and Wilhelm were using a Leiden scale, but incorrectly. This could have come about had they adopted the nbp of $-252.754^{\circ} \mathrm{C}$ reported by Keesom, Bijl, and Van der Horst [18] combined with the as sumption, $0^{\circ} \mathrm{C}=273.16^{\circ} \mathrm{K}$. This would have been erroneous because Keesom et al. had based their $\mathrm{nbp}$ on $0^{\circ} \mathrm{C}=273.13^{\circ} \mathrm{K}$. If this were the source of Johns and Wilhelm's scale, then a translational shift by -0.03 deg would be in order. I have not found any other ways of accounting for their assumed value for the nbp. Either way of correcting their scale changes the specific refraction by only a few parts in $10^{4}$. The difference between the two ways just outlined is at most 1 part in $10^{4}$ in $r$. This is insignificant, inasmuch as their claimed accuracy of 0.0003 in $n$ corresponds to about \pm 24 parts in $10^{4}$ in $r$.

The corrected results of Johns and Wilhelm are given in figures 1 and 2 and Table 2. The three points in figure 2 show downward 
curvature, the same defect noted in the data of Belonogov and Gorbunkov. Hence, the dispersion formula is again truncated after the term in $1 / \lambda^{2}$.

3.3. Data of Augustin [3]

These were determined at five wavelengths for saturated liquid at $745.52 \mathrm{~mm}$ pressure. Using modern vapor pressure data [19], the temperature is found to have been $20.316^{\circ} \mathrm{K}$. The determination was made by measuring critical angles. The experiment was very similar to that of Johns and Wilhelm. However, because of the way in which the optical cell was constructed, the measurements gave the ratio of the refractive indices of saturated liquid and vapor, whereas the refractive indices by Johns and Wilhelm were referenced to vacuum. Augustin obtained the refractive indices of the liquid from the above ratios by using estimated values of the refractive index of the vapor. He obtained these by assuming the L-L ratio to be constant between STP and the saturated vapor at $20.3^{\circ} \mathrm{K}$ and by assuming the vapor to be ideal. The saturated vapor density that he calculated can now be seen to be $6 \%$ low. Although experimental refractive indices of the saturated vapor are still unavailable, this part of Augustin's procedure can now be improved by using certain modern data. In addition we can calculate $\mathrm{n}_{\lambda}$ at all five wavelengths, whereas Augustin had to omit one wavelength for lack of subsidiary data. Two procedures can be suggested:

(a) Starting with experimental data for $\mathrm{n}_{\lambda}$ of the gas at STP, such as those described in sec. 2, calculate $r_{\lambda}$ from the L-L formula (2) at the wavelengths used by Augustin. Assume that these values are valid for the saturated vapor at $20.3^{\circ} \mathrm{K}$. Using modern data for the density of the saturated vapor, convert the $r_{\lambda}$ values to $n_{\lambda}$ values for the saturated vapor using the $L-L$ formula (2).

(b) Start with the value of specific polarization at the density of the saturated vapor calculated from Stewart's formula (eq. 8 in 
sec. 4). Equate this to $r_{\infty}$ by Maxwell's relation (4). Use the accurate formula (7) for dispersion of the gas at STP to convert this to values of $r_{\lambda}$ at the desired wavelengths. The final step is the same as in procedure (a).

Procedure (a) requires only optical data but assumes that the L-L ratio is constant over a very wide range of temperatures. Procedure (b) only requires the relatively small dispersion corrections to be constant over this same range. Hence, (b) is to be preferred and was used.

The density of the saturated vapor was found from the recent correlation by Stewart and Roder [19]. The specific polarization at this density was calculated to be $1.00439 \mathrm{~cm}^{3} / \mathrm{g}$. The values of $\mathrm{n}_{\lambda}$ for the vapor and, hence, those calculated for the liquid, are as much as $0.02 \%$ higher than Augustin's. The corrected values of $r_{\lambda}$ are given in figures 1 and 2 and Table 2 .

Augustin's paper appears to be a careful and precise work. This impression is supported by the appearance of figures 1 and 2 , including the fact that the curvature in figure 2 is of physically realistic sign. Hence the three-term dispersion formula (7) could be used to fit his data. Another evidence of the quality of this work is the fact that the saturated liquid density as determined by Augustin in a separate experiment is within $0.06 \%$ of modern data [14].

\subsection{Data of Scheel [20]}

The refractive index of the gas at $1 \mathrm{~atm}$ pressure at two temperatures in the liquid air range as well as at STP was determined. The low temperature data, if accurate enough, would be of interest as midpoints in tracing the slight temperature variation of the L-L ratio from room temperature to the $20^{\circ} \mathrm{K}$ region. Unfortunately the data of Scheel for the gas at STP are substantially lower than the data from the five 
sources reviewed in sec. 2. The difference approaches $1 \%$ in (n - 1). Because of this evidence of systematic error, the low temperature data of Scheel were not used.

\subsection{Summary and Comparison of the Data}

Figures 1 and 2 and Table 2 compare four sets of specific refractions for normal hydrogen with one set for parahydrogen. Of the data for the liquid, those of Augustin are thought to be the most accurate, those by Belonogov and Gorbunkov the least accurate, and those by Johns and Wilhelm intermediate but not much more accurate than those of Belonogov and Gorbunkov. The data for the STP gas from $\mathrm{K}-\mathrm{K}$ are based on much more accurate data; however, it is a moot question how well they represent the specific refraction of the liquid.

4. Calculation of Refractive Index from Dielectric Constant In outline, the calculation proposed in the Introduction involves the following steps:

(a) From the P-p-T correlation of Goodwin et al. [21] find the density of the desired state.

(b) From Stewart's formula

$$
\frac{1}{p}=0.99575-0.09069 \rho+1.1227 p^{2} \quad, g-c m \text { units }
$$

calculate the specific polarization.

(c) Equate this to $r_{\infty}$ by Maxwell's identity (4). (Recent accurate measurements of the dielectric constants of several gases by Dunn [22] confirm the validity of this identity for substances like hydrogen which do not show anomalous dispersion.)

(d) Calculate the specific refraction at the desired wavelength by the Cauchy-type dispersion formula (7).

(e) Extract the refractive index value from the L-L formula (2). 
Stewart's formula (8) is for fluid parahydrogen and is limited to temperatures below $100^{\circ} \mathrm{K}$ and densities below $0.080 \mathrm{~g} / \mathrm{cm}^{3}$.

This method of calculation yields specific refractions that depend on density. Figure 1 contains two illustrative curves calculated for densities at which the practical extrema of $r_{\lambda}$ occur. Also, in order to find out how well this calculation represents the experimental data, the refractive index was calculated at each coordinate $(T, \lambda)$ at which a refractive index value has been reported for the (saturated) liquid. The results are summarized in Table 3. It will be seen that the agreement is comparable to the agreement of the experimental investigations among themselves. This comparison was made without applying any corrections for the para-normal difference. If the para-normal difference found by Belonogov and Gorbunkov could be assumed to apply under all conditions, the deviations listed in Table 3 for Johns and Wilhelm and for Augustin would all be increased by 6 units.

Table 3

Summary of deviations, $10^{4}$ ( $\mathrm{n}_{\text {calc. }}{ }^{-n_{\text {exptl. }}}$ ) The calculated values of refractive index of liquid hydrogen were obtained by the method of sec. 4. Comparisons were made without regard to ortho-para composition.

\begin{tabular}{|c|c|c|c|c|c|c|c|}
\hline \multirow{2}{*}{$\begin{array}{l}\lambda \\
\AA\end{array}$} & \multicolumn{3}{|c|}{$\begin{array}{c}\text { Belonogov \& Gorbunkov } \\
\text { para }-\mathrm{H}_{2}\end{array}$} & \multicolumn{3}{|c|}{$\begin{array}{c}\text { Johns \& Wilhelm } \\
\text { normal- } \mathrm{H}_{2}\end{array}$} & \multirow{2}{*}{$\frac{\begin{array}{r}\text { Augustin } \\
\text { normal-H }\end{array}}{\text { Dev.* }}$} \\
\hline & $\begin{array}{l}\text { Avg. } \\
\text { Dev. }\end{array}$ & $\begin{array}{l}\text { Max. } \\
\text { Dev. }\end{array}$ & $\begin{array}{l}\text { No. of } \\
\text { points }\end{array}$ & $\begin{array}{l}\text { Avg. } \\
\text { Dev. }\end{array}$ & $\begin{array}{l}\text { Max. } \\
\text { Dev. }\end{array}$ & $\begin{array}{l}\text { No. of } \\
\text { points }\end{array}$ & \\
\hline 4047 & & & & & & & -1.1 \\
\hline $4358-60$ & -8.0 & -15 & 7 & -3.6 & -7 & 8 & -0.6 \\
\hline $5460-61$ & +0.1 & +5 & 7 & -0.9 & -7 & 9 & -0.1 \\
\hline 5790 & +2.5 & +8 & 7 & & & & -0.5 \\
\hline 6563 & & & & & & & -1.3 \\
\hline 6939 & & & & +3 & +4 & 2 & \\
\hline Wtd. Avg. & -1.8 & & & -1.6 & & & -0.7 \\
\hline
\end{tabular}

* One experimental point at each wavelength 


\section{The Ortho vs Para Difference}

The comparison between normal and parahydrogen at $5460 \AA$ by Belonogov and Gorbunkov, if applicable at all wavelengths, would permit one to adjust all of the data to the same basis of o-p composition. Thus, in figure 1 the points of Johns and Wilhelm and of Augustin and in figure 2 these plus the $\mathrm{K}-\mathrm{K}$ curve would be lowered relative to the others by the length of the arrow in each figure. Unfortunately, such an adjustment would worsen the overall agreement. This result, taken with the various deficiencies of the work of Belonogov and Gorbunkov that have been pointed out, suggests that their value for the normal-para difference should not be accepted in the absence of confirmation.

This conclusion is supported by the result of the following theoretical calculation in which we estimate the polarizability difference, $\alpha_{1}-\alpha_{0}$, between the ortho and para hydrogen ground states; i.e., $\mathrm{J}=1$ and $J=0$, respectively. Since the polarizability is proportional to the specific polarization according to the relation [13],

$$
\alpha=3 \mathrm{Mp} / 4 \pi \mathrm{N}
$$

we may assume, neglecting dispersion, that the calculated percentage difference in $\alpha$ should correspond to the observed percentage difference in $r_{\lambda}$.

We begin by calculating the centrifugal stretching in the $\mathrm{J}=1$ state. The centrifugal force balance is described by the following equation [23]:

$$
K\left(a_{1}-a_{0}\right)=P^{2} / \mu a_{1}{ }^{3}
$$


The angular momentum is given by quantum theory as

$$
P=(J[J+1])^{1 / 2} \mathrm{~h} / 2 \pi
$$

and the reduced mass $\mu$ is just $\mathrm{m} / 2$. Hence

$$
a_{1}-a_{0}=h^{2} / \pi^{2} K m a_{1}^{3}
$$

From this formula the stretching $a_{1}-a_{0}$ is found to be $1.12 \times 10^{-3} \AA$ or $0.15 \%$ of the internuclear distance, $a_{0}$. From this value and an estimate of the coefficient $\mathrm{d} \alpha / \mathrm{da}$, we can calculate the polarizability difference.

There have been several calculations of $\mathrm{d} \alpha / \mathrm{da}$ according to various theoretical models. Values in the range, $0.89-1.49(\AA)^{2}$, have resulted. These have been summarized by Ishiguro et al. [24], whose value 1.411 we adopt. In addition, Bell and Long [25] list two "experimental" values: 0.67 from the relative degrees of depolarization of Rayleigh and Raman scattering--regarded by them as inaccurate--and 1.68 from the isotope effect, calculated as $\left(\alpha_{H}-\alpha_{D}\right) /\left(a_{H}{ }^{-a_{D}}\right)$. An alternate calculation will be made based on the latter value which gives the highest of the calculated values of polarizability difference. Thus, for $\mathrm{d} \alpha / \mathrm{da}=1.411$ (Is higuro) we obtain $\alpha_{1}-\alpha_{0}=1.58 \times 10^{-3}(\AA)^{3}$ or $0.20 \%$ of $\alpha$. For $\mathrm{d} \alpha / \mathrm{da}=1.68$ (Bell) we obtain $\alpha_{1}-\alpha_{0}=1.88 \times 10^{-3}(\AA)^{3}$ or $0.24 \%$ of $\alpha$.

The normal-para difference found by Belonogov and Gorbunkov from the refractive indices was $(0.6 \pm 0.1) \%$ of $r_{5460 \AA}$. The orthopara difference would be one-third greater and thus at least three times as large as any of the theoretically calculated differences. 


\section{Proposed Experimental Method}

It is sometimes suggested that it is more accurate to derive dielectric constants from refractive indices by the Maxwell relation, $\epsilon=\mathrm{n}_{\infty}^{2}$, than to determine them directly. This is undoubtedly true for gases because the refractive index can be determined by very sensitive interferometric methods. However, the opposite is likely to be true for liquids, as this study exemplifies, because of the custom of using less accurate deviation methods for the refractive index. Interferometers have apparently not been used for absolute measurements on liquids because of the impossibility of counting the passage of fringes continuously while is othermally attenuating the sample to a vacuum; i.e., the discontinuity at the liquid-vapor phase change would destroy the continuity of the fringe count. Yet it is obvious that continuity could be maintained in principle by following a path in the $\mathrm{P}-\mathrm{T}$ plane that would pass above the critical point and not intersect the coexistence line. Thus, in the schematic diagram, to get from $A$ to $E$ without crossing the coexistence curve, one could follow the path ABCDE. Considerable care would have to be taken to maintain homogeneity of density in the optical cell during such a change; otherwise the fringe pattern would be lost.

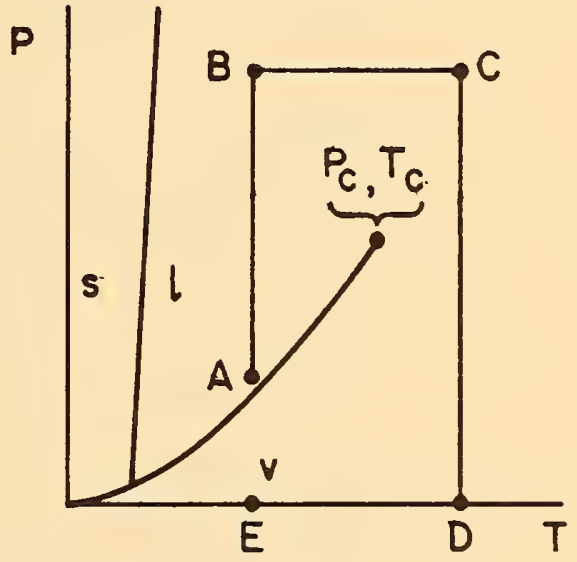
Use of a Fabry-Perot etalon with equalization of the inside and outside pressures is attractive, inasmuch as distortions due to pressure are then limited to the small and calculable effect of hydrostatic compression of the spacer. Sensitivity of the order of $1 \times 10^{-6}$ in $\mathrm{n}$ with etalon length as short as $1 \mathrm{~cm}$ appears to be feasible. 


\section{Symbols}

a

K

$\mathrm{m}$

$\mathrm{n}$

$\mathrm{n}_{\infty}$

$\mathrm{p}$

P

r

$r_{\infty}$

$\alpha$

$\epsilon$

$\lambda$

$v$

$\rho$

$\mu$

internuclear distance in diatomic molecules. (Subscripts refer to rotational quantum numbers. A rounded value for $\mathrm{H}_{2}$ is $0.74 \AA_{\text {。 }}$ )

rotational quantum number

force constant for internuclear stretching. For $\mathrm{H}_{2}$ its value is $0.57 \times 10^{-10} \mathrm{erg} /(\AA)^{2}[23]$.

atomic mass

refractive index

refractive index extrapolated to infinite wavelength specific polarization, $(\epsilon-1) / \rho(\epsilon+2)$

angular momentum

specific refraction, $\left(n^{2}-1\right) / \rho\left(n^{2}+2\right)$

specific refraction at infinite wavelength

polarizability, $3 \mathrm{Mp} / 4 \pi \mathrm{N}$. A rounded value for $\mathrm{H}_{2}$ is $0.79(\AA)^{3}$.

dielectric constant

wavelength

frequency

density

reduced mass of molecule 


\section{References}

[1] A. V. Belonogov and V. M. Gorbunkov, Optics and Spectroscopy 14, 234 (1963). [Original in Optika i Spektroskopiia 14, 438 (1963)].

[2] H. E. Johns and J. O. Wilhelm, Can. J. Res., 15A, 101 (1937).

[3] H. Augustin, Ann. d. Physik 46, 419 (1915).

[4] J. W. Stewart, J. Chem. Phys. 40, 3297 (1964).

[5] Landolt-Börnstein Tables, 6th ed., Vol. II, Part 8 (1962) pp. 872, $879,882,885,887$.

[6] A. A. Maryott and F. Buckley, Table of Dielectric Constants and Electric Dipole Moments of Substances in the Gaseous State, NBS Circular 537 (1953), p. 3.

[7] C. Cuthbertson and M. Cuthbertson, Proc. Roy. Soc. (London), A83, 151 (1909).

[8] J. Koch, Ark. Mat. Astr. Fys. 8, No. 20 (1912).

[9] M. Kirn, Ann. Physik 64, 566 (1921).

[10] J. Tausz and H. Görlacher, Z. tech. Phys. 12, 19 (1931).

[11] O. E. Frivold, O. Hassel, and S. Rustad, Phys. Z. 38, 191 (1937).

[12] L. Essen, Proc. Roy. Soc. (London), B66, 189 (1953).

[13] E. U. Condon and H. Odishaw, editors, Handbook of Physics, 1958, p. 6-111 (See also p. 6-109 et seq. and p. 4-111 et seq.) (McGraw-Hill Book Co., Inc., New York, N. Y.).

[14] R. D. Goodwin, D. E. Diller, H. M. Roder, and L. A. Weber, Cryogenics 2, 81 (1961).

[15] H. Kamerlingh Onnes and C. A. Crommelin, Leiden Commun. 137 a (1913). 
[16] G. W. Moessen, J. G. Aston, and R. G. Ascah, J. Chem. Phys. 22, 2096 (1954).

[17] F. Henning and W. Heuse, Z. Physik 23, 105 (1924).

[18] W. H. Keesom, A. Bijl, and H. Van der Horst, Leiden Commun. No. 217a (1931).

[19] R. B. Stewart and H. M. Roder, Chapter 11 in Technology and Uses of Liquid Hydrogen, edited by Scott, Denton, and Nicholls (Pergamon Press, Oxford, 1964).

[20] K. Scheel, Verhandl. deut. physik. Ges. 9, 24 (1907).

[21] R. D. Goodwin, D. E. Diller, H. M. Roder, and L. A. Weber, J. Research NBS, 67A, 173 (1963).

[22] A. F. Dunn, Can. J. Phys. 42, 1489 (1964).

[23] G. Herzberg, Spectra of Diatomic Molecules, 2nd edition, 1950, pp. 104, 458 (D. Van Nostrand Co., Inc., Princeton, N. J.)

[24] E. Ishiguro, T. Arai, M. Kotani, and M. Mizushima, Proc. phys. Soc. (London), A65, 178 (1952).

[25] R. P. Bell and D. A. Long, Proc. Roy. Soc. (London), A203, 364 (1950). 


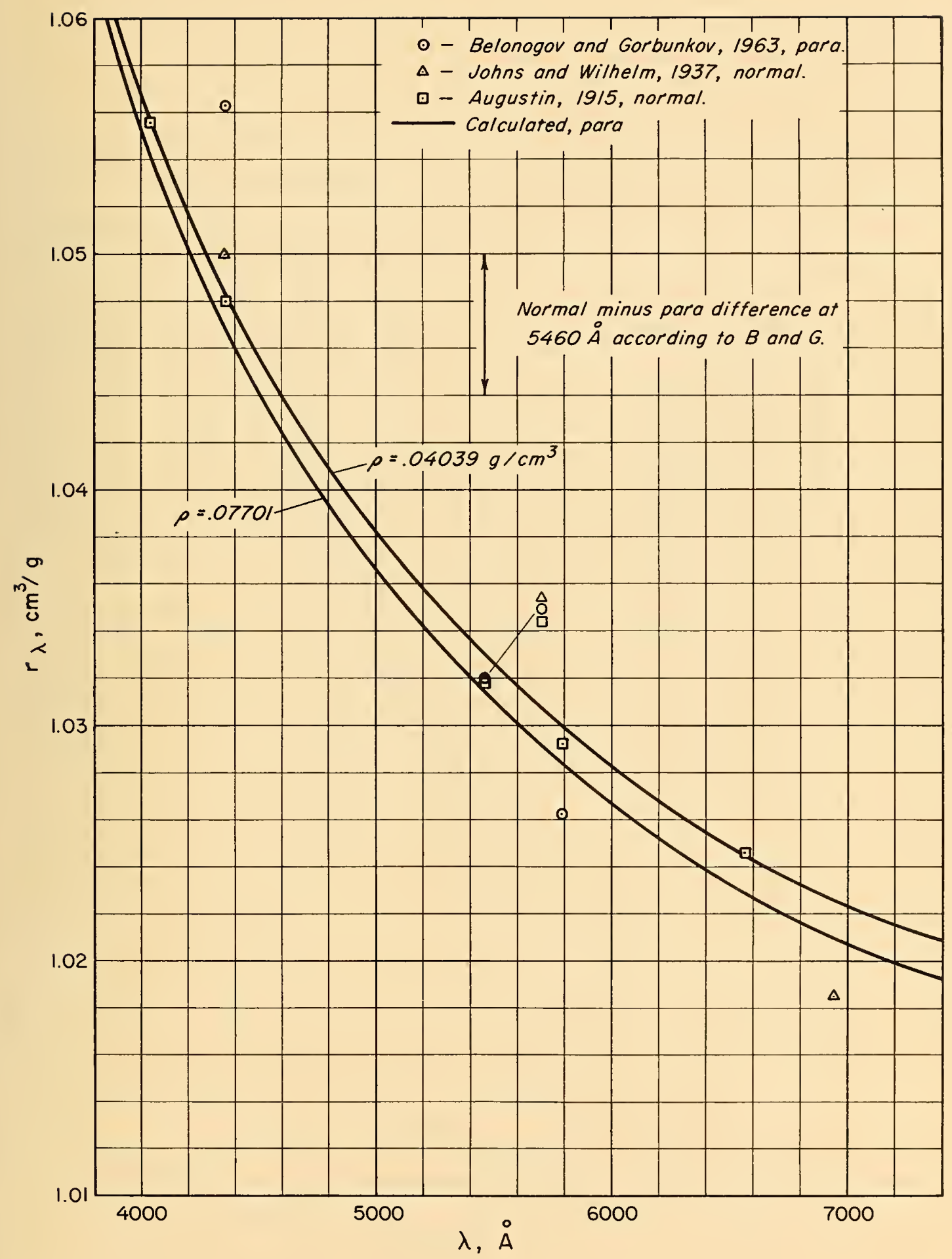

Figure 1. Specific refraction of liquid hydrogen as a function of wavelength.

The two curves show the extremes given by the method of sec. 4 for saturated liquid. The lower one is for the triple point density. The upper one is for the density at which $\mathrm{p}$ and $\mathrm{r}$ are maximum. This is near to the critical density. With further decrease of density, $p$ and $r$ decrease again towards the lower curve. (See figure 4 of the paper by Stewart [4] for the density variation of p.) 


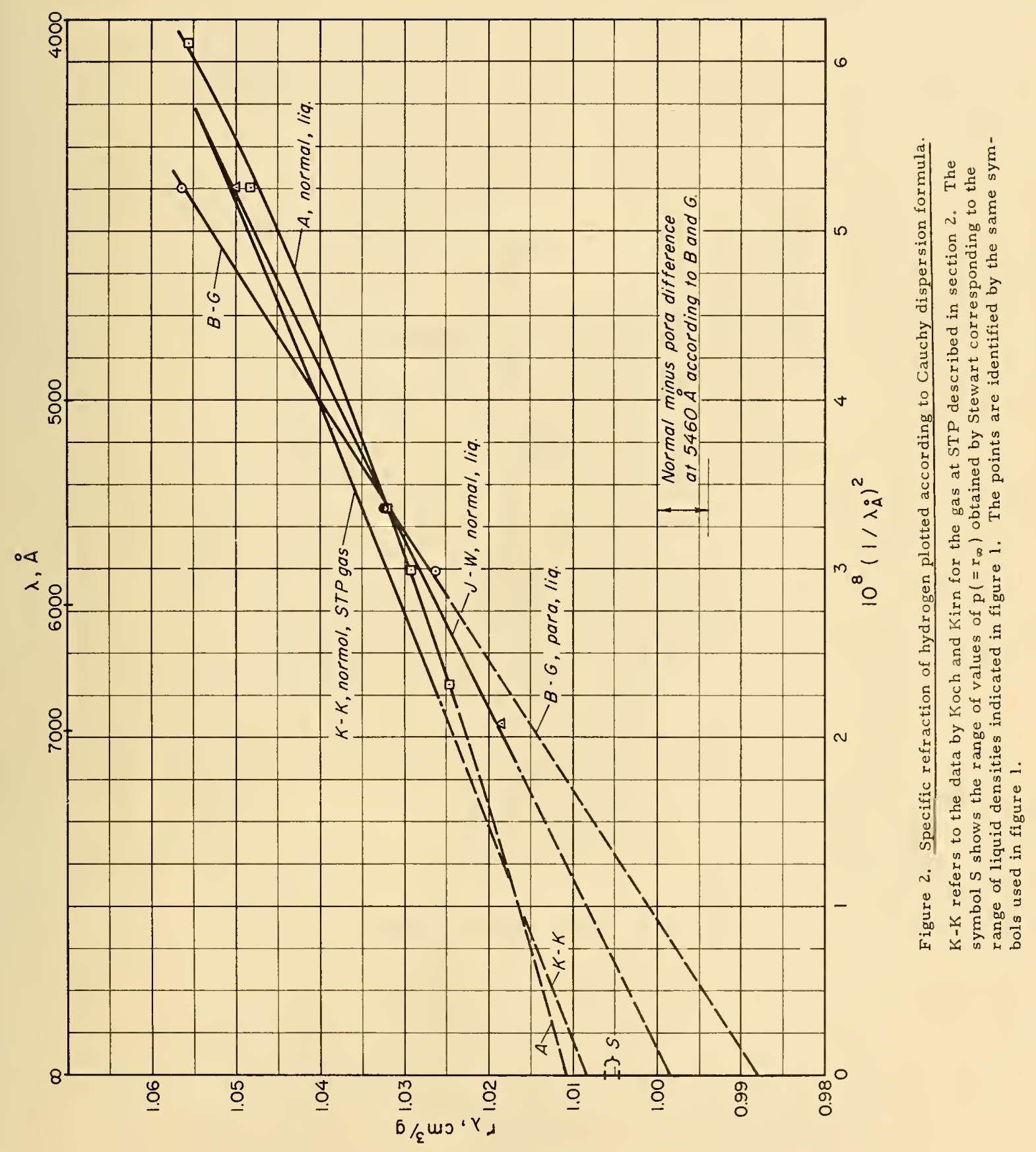


